\title{
Study of Isohyets Generation Algorithm and Its Modification
}

\author{
Mei-Ling Yin \\ Hubei Province Key Laboratory of Intelligent Information \\ Processing and Real-time Industrial System \\ Wuhan University of Science and technology \\ Wuhan, China \\ E-mail: 1091635077@qq.com
}

\author{
Jian-Xun Chen \\ Hubei Province Key Laboratory of Intelligent Informat ion \\ Processing and Real-time Industrial System \\ Wuhan University of Science and technology \\ Wuhan, China \\ E-mail: 7500080@qq.com
}

\begin{abstract}
Generally speaking, isohyets need to be generated through triangulated mesh, linear interpolation, search contours. Triangulated mesh is the key step, it is especially critical to improve its efficiency and reduce its time complexity, and its prevalent two generation algorithms are divide-conquer and incremental insertion algorithms, after study on the compound algorithm which is based the two mentioned ones. In this paper, we posed a high-efficiency-compound algorithm, by searching boundary before the triangulation mesh, and by reducing the number of the nearest neighbor points in the triangulation, we can determine whether the angle is the maximum. This thesis improves on the common algorithm, and the algorithm is implemented in the analysis of rainfall in Gongan County, Hubei Province. We can reduce the complexity of computation, and improve the efficiency of the isohyets generation algorithm.
\end{abstract}

Keywords-isohyets; triangulation; mesh; contours

\section{INTRODUCTION}

The isohyetose is a line on which all of points have equal rainfall, it was drew according to the once rainfall or period rainfall obtained from the meteorological station. It is important reference for all levels of government and flood prevention departments to make decision, owing to its visuality and legibility [1].With the rapid development of computer technology, and drawing of isoline has been applying to a wide range of areas, particularly in the field of Flood Control System, isoline map has been wide applied chart. The so-called isoline map drawing is the process of interpolation with mathematical methods and converting points with the same value into chart, using large numbers of discrete and regular physics or geometry data in two-dimensional plane .It is the combination of data and figure, so that technical staff can easily find the trends of changing data, to get the results of computer simulation intuitively. As a method to reflect value change, isoline was widely used in modern scientific research, particularly in the flood control system this method is widely used. Through isoline map, we can have a very intuitive understanding of real-time rainfall information.

\section{RELATED WORK}

There are many isoclines rendering algorithms at the present time, including discrete data interpolation and fitting, Delaunay triangulation meshing, calculation of equivalent points, contour tracking, abnormal control in the tracking, the filling of the regional equivalent and so on. Triangulated mesh is the key, divide-conquer, incremental and grow method of triangular network.

The Research of the algorithms for triangulated mesh is relatively early. B.P.Heap, McLain D-H [2] proposed algorithms for the production of contour map over an irregular triangular mesh. Peraire [3] proposed an adaptive mesh procedure for computing steady state solutions of the compressible ruler equations in three dimensions. Ey mard[4] proposed appropriate constraints on finite-element grids and convenient definitions for volumes and transmissibility leads to a convergence property for the CVFE scheme. Joe [5, 6] and Weatherill[7] proposed various improvement methods of delaunay partition. Domestic studies are mainly focus on the depth or imp rovement of the algorithm of contour lines. Yi Li proposed delaunay triangulation meshing and illustrated interpolation in meshes and contour tracing [8]. The method improves the precision of the precipitation isoline and is important to count the surface precipitation of the drainage area. Huang Binghu, Sun Genyun proposed a new method of interpolating urban rainfall monitoring stations and to extract isohyets. The automatic extracting steps of isohyets is analyzed, and based on ArcGIS engine giving the hey codes and main ideas[9].Peng Siling, Deng Min implement three basic interpolation methods in ArcEngine provides by ESRI, which are IDW, Spline and Kriging. Precipitation data obtained from 31 rainfall stations in Jinan are employed to draw the rain isoline with the three interpolation method, and the interpolation results of these three methods are compared[10].The analysis of rainfall contours depends on the artificial, which leads to great labor intensity and low efficiency. At the same time, using some special drawing contour software such as Surfer, although it can draw the contour line, but it is embedded into the soft. The system method is not flexible enough, and the function is single, the data input is extremely tedious, and some large GIS software such as MapInfo, ArcInfo and so on, although it also provides the contour line drawing function, but the required data are artificial input, contour map into the degree of automation is not high.

In this study, we proposed a novel method to track is ohyets, the contributions of the work include:

1) Convex hull scanning algorithm is used to search boundary before the triangulation mesh, which can improve the efficiency. 
2) During the triangulation mesh, the discrete points are divided into the left and right, which reduce the number of finding nearest neighbors, and store the equivalent point in a collection.

\section{THE IMPROVED METHOD}

\section{A. Searching Boundary}

The boundary of data points must be obtained for triangulation of scattered data points. In order to improve the work efficiency, we use Convex Hull scanning algorithm.

There may be a concave boundary as shown in figure 1 below. The convex hull $\Omega$ of discrete points $P\left\{p_{1}(x, y), p_{2}(x, y), \ldots p_{n}(x, y)\right\}$ is a minimum convex polygon; to make all points in the $\mathrm{P}$ is on the edge of a polygon or within it. The convex hull algorithm's purpose is to find the minimum convex polygon, named the boundary of data points. The common convex hull algorithms are divide-and-conquer, fast packet method, incremental algorithm, Graham scanning method and Jarvis forward method. The time complexity of these algorithms is from $O\left(\right.$ nlnn) to $O\left(n^{2}\right)$. Not all the collected data sets are convex; there may be a concave boundary, the convex hull algorithm cannot identify the concave boundary. The corresponding method is to divide the data points; we can get many pieces of convex hull. To obtain boundary for each sub convex, stitching the sub boundaries by counter clockwise. The entire boundary of the data point set is obtained: $\Gamma=\Gamma_{1} \cup \Gamma_{2} \cup \ldots \cup \Gamma_{n}$.

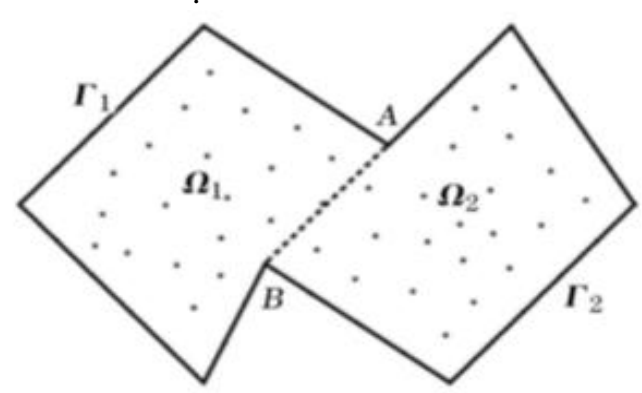

Figure 1. Block search for the concave boundary of scattered points.

\section{B. Building Triangulated Irregular Network}

Using triangulated irregular network for expressing the digital elevation model can not only reduce the data redundancy caused by the regular grid method, but also the computation efficiency is superior to the pure line based method. Take a point from all data points, and find the closest point to this point, the three points will be the first triangle. Based on the three sides of the first triangle, the point of satisfying condition is sought in three different directions and formed a new triangle. On the basis of the new triangle to spread around, until the entire area is filled with triangles.

There are only two edges of each triangle to expand, on the base of the baseline, judges the nearest neighbor points and the discrete points are divided into the left and right. Use the required set of points to judge, which reduce the number of finding nearest neighbors, it only needs to determine whether the angle is the largest, do not need to judge whether it is located in the left or right. This represents great improvement when compared with other introduced. It is worth mentioning that the D-TIN data structure designing brings in traditional data structures, which is based on PointClass and LineClass provided by ArcEngine as the basis to add some new properties. Respectively defined CoordinatePoint, Edge and Triangle. Puts forward a new structure to store points and lines, it is more conducive to the calculation of the equivalent point and contour tracing. To compare the efficiency of the improved algorith $m$ and the pre algorith $\mathrm{m}$, we randomly generated discrete points. The time of triangular mesh is as Table I.

TABLE I. TABLE THE COMPARISON OF THE EFFICIENCY BEFORE AND AFTER THE IMPROVED ALGORITHM

\begin{tabular}{|c|c|c|c|c|}
\hline $\begin{array}{c}\text { Discrete } \\
\text { points }\end{array}$ & Edges & Triangles & $\begin{array}{c}\text { Time before } \\
\text { improvement(s) }\end{array}$ & $\begin{array}{c}\text { Time after } \\
\text { improvement(s) }\end{array}$ \\
\hline 1000 & 2.918 & 1.969 & 1.33 & 0.14 \\
\hline 2000 & 5.839 & 3.930 & 5.97 & 3.23 \\
\hline 3000 & 8.689 & 5.827 & 13 & 4.87 \\
\hline 5000 & 14.341 & 9.742 & 39.04 & 8.98 \\
\hline 7000 & 20.190 & 13.591 & 81.98 & 11.6 \\
\hline 15000 & 44.039 & 29.127 & 396.09 & 13.4 \\
\hline 20000 & 38.963 & 59.085 & 791.60 & 14.8 \\
\hline 50000 & 148.677 & 98.683 & 2168.72 & 16.09 \\
\hline
\end{tabular}

It can be seen from Table 1 that the efficiency of the improved algorithm is greatly improved, and the effect is more obvious when the number of discrete points is more. Compared with the literature [1.4], the efficiency of the improved algorithm is also improved, which shows that it is feasible to improve the algorithm of triangulation.

\section{Calculation Of Contour Lines}

We often use discrete point data as the bas is for contour tracking. Before drawing the contour, we should construct a regular grid or irregular grid in the area covered by the discrete points. Then the contour lines can be generated on the basis of regular or irregular grids.

For any triangular mesh, set the three vertex numbers are $\mathrm{I}, \mathrm{J}, \mathrm{K}$. The three vertex coordinates are denoted as $\left(\mathrm{x}_{1}, \mathrm{y}_{1}, \mathrm{z}_{1}\right)\left(\mathrm{x}_{2}, \mathrm{y}_{2}, \mathrm{z}_{2}\right)\left(\mathrm{x}_{3}, \mathrm{y}_{3}, \mathrm{z}_{3}\right) . \mathrm{h}$ is the pre drawn contour of the elevation value.

If the contour line passes through the edge, then the intersection coordinates $\left(\mathrm{x}_{0}, \mathrm{y}_{0}, \mathrm{z}_{0}\right)$ can be got by interpolation:

$$
\left\{\begin{array}{l}
x_{0}=x_{1}+\left(x_{2}-x_{1}\right) *\left(h-z_{1}\right) /\left(z_{2}-z_{1}\right) \\
y_{0}=y_{1}+\left(y_{2}-y\right) *\left(h-z_{1}\right) /\left(z_{2}-z_{1}\right) \\
z_{0}=h
\end{array}\right.
$$




\section{Contour Tracing}

1) Non-closed contour tracing

- We ground on the numerical value of a contour, to find an equivalent edge at the boundary. If we can't find the starting edge, which shows that the contour line is closed, Step (2). The edge is recorded to the equivalent edge series. And then remove it from the line series to be searched.

- Search for the second equal edge in the related triangle, added to the equivalent edge series after finding it. And then remove it from the line series to be searched.

- Remove the triangle from the series of triangles to be searched; the newly found equivalent edge is only one related triangle. Search the next equivalent edge in the triangle, added to the equivalent edge series after finding it, and then remove it from the line series to be searched.

- Recursive execution (3) until the last equivalent boundary is found.

- A non -closed contour line search is completed, as shown in figure 2 below.

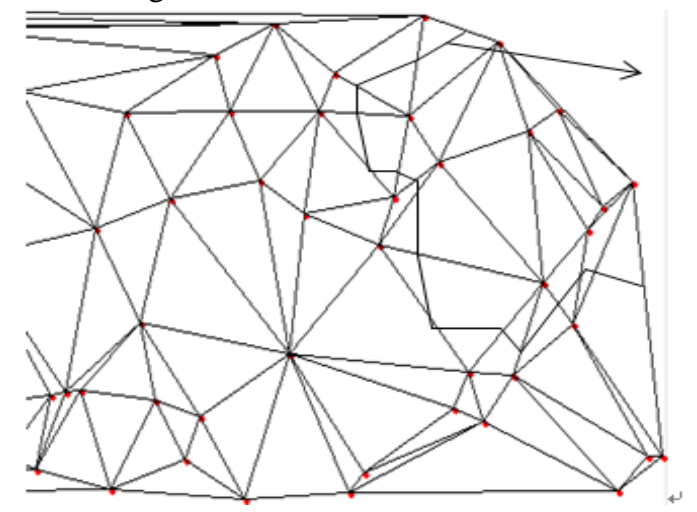

Figure 2. Non-closed contour.

\section{E. Smoothing The Contour Lines}

The result of contour tracing is to get the equivalent point from the network, and then connect these contour points in turn with the line segment. At this time the obtained contour is not smooth, and the curve of the contour is determined by the size of the grid. When the grid size is large, although the results obtained can be expressed by the degree of vertical variation of the terrain, but it has affected people's aesthetic effect. Therefore, it is necessary to smooth the contour map of the tracking. The tension spline function method is used to carry out the smoothing process. The basic idea is that the piecewise interpolation function is a linear combination of linear interpolation and two hyperbolic functions $s h \sigma x$ and $\operatorname{ch} \sigma x$

$$
f(x)=c_{1}+c_{2} x+c_{3} \operatorname{sh} \sigma x+c_{4} \operatorname{ch} \sigma x
$$

\section{2) Closed contour tracing}

- Looking for an equivalent edge as the start edge. if we can't find it, it shows that we can't analyze the contour line. Stop the analysis and start to search for another contour line. And then record the edge to the equivalent edge series. Finally make a mark on the edge.

- Search for the second equal edge in the related triangle, added to the equivalent edge series after finding it. And then remove it from the line series to be searched.

- Remove the triangle from the series of triangles to be searched, the newly found equivalent edge is only one related triangle. Search the next equivalent edge in the triangle, added to the equivalent edge series after finding it, and then remove it from the line series to be searched.

- Recursive execution (3) until the first equivalent boundary is found.

- A closed contour line search is completed, as shown in figure 3 below.

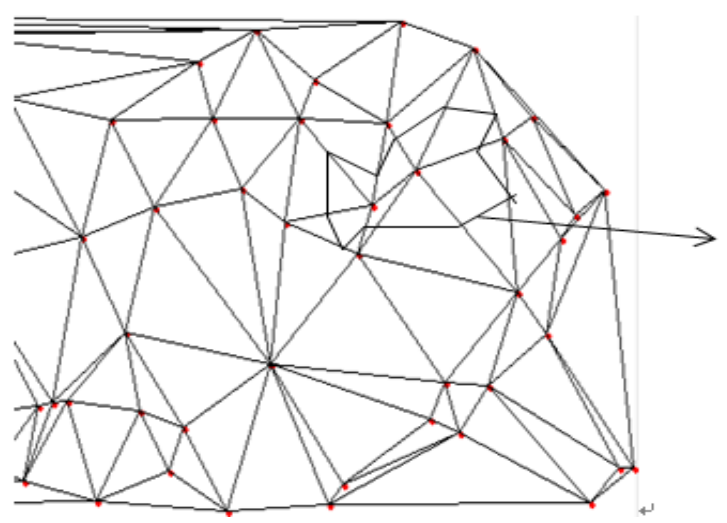

Figure 3. Closed contour.

Equation (1) has continuous second derivative, $\sigma$ play a role in controlling the position of the inflection point and the shape of the curve. Through complex mathematical derivation, the function expression of the tension spline function can be obtained:

$$
\begin{aligned}
& f(x)=\frac{1}{\sigma^{2} \operatorname{sh}\left(\sigma \mathrm{h}_{i}\right)}\left[f^{\prime \prime}\left(x_{i}\right) \operatorname{sh}\left(\sigma\left(\mathrm{x}_{i+1}-\mathrm{x}\right)\right)+f^{\prime \prime}\left(\mathrm{x}_{i+1}\right) \operatorname{sh}\left(\sigma\left(\mathrm{x}-\mathrm{x}_{i}\right)\right)\right] \\
& +\left[y_{i}-\frac{f^{\prime \prime}\left(x_{i}\right)}{\sigma^{2}}\right] \frac{x_{i+1}-x}{h_{i}}+\left[y_{i+1}-\frac{f^{\prime \prime}\left(\mathrm{x}_{i+1}\right)}{\sigma^{2}}\right] \frac{x-x_{i}}{h_{i}}
\end{aligned}
$$

$$
x_{i} \leq x \leq x_{i+1}(\mathrm{i}=0,1,2, \cdots, \mathrm{n}-1) ; \mathrm{h}_{i}=x_{i+1}-x_{i}
$$

If $\sigma \rightarrow 0$, then

$$
f^{\prime \prime}(x)=f^{\prime \prime}\left(x_{i}\right) \frac{x_{i+1}-x}{h_{i}}+f^{\prime \prime}\left(x_{i+1}\right) \frac{x-x_{i}}{h_{i}}
$$


The above formula is a standard three spline function expression. The three spline function can ensure that the curve is smooth, and it can also be used as a data node.

$$
\begin{aligned}
& \text { If } \sigma \rightarrow+\infty \text {, then } \\
& \qquad f(x)=y_{i} \frac{x_{i+1}-x}{h_{i}}+y_{i+1} \frac{x-x_{i}}{h_{i}}
\end{aligned}
$$

At this time, the tension spline function is reduced to a piecewise linear function. Piecewise linear function can absolutely guarantee that the line segment does not intersect.

The algorithm is simple and the efficiency is relatively high, the contour is completely through the equivalent point, and the lines are smooth.

\section{EXPERIMENTAL RESULT}

In order to verify the feasibility of the algorithm, after generating delaunay triangulation, calculation of contour lines, contour tracing and smoothing the contour lines, otching up rainfall contours of AnGong Hubei in September 12,2016 . The is ohyet chart is as shown in figure 4 below:

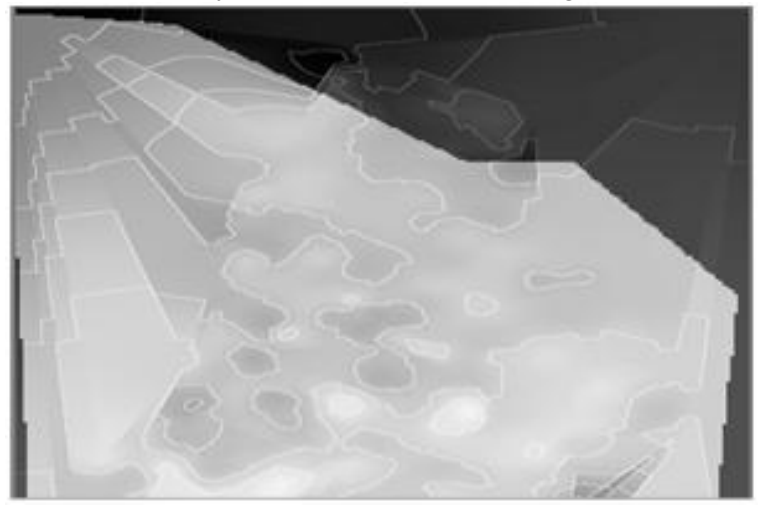

Figure 4. Rainfall contours of AnGong Hubei in September 12, 2016

\section{CONCLUSION}

This study clearly demonstrates the generation algorithm of is ohyets, and uses examples to demonstrate the feasibility of the algorithm. By searching boundary before the triangulation mesh, and reducing the number of the nearest neighbor points in the triangulation. The results show that the proposed method can improve the efficiency and reduce the redundancy of code. The method improves the precision of the precipitation is oline and is important to count the surface precipitation of the drainage area.

\section{REFERENCES}

[1] Liu Jun, Li Shunxin, Chen Jianxun. Research and Implementation of Generation of Equal rainfall lines based MapX.YANGTZE RIVER,2005,36(4):234 242.

[2] D.H.McLain. Drawing contours from arbitrary data points. The Computer Journal, 1974(17):316 324

[3] J Peraire, M Vahdati, K Morgan, O.C Zienkiewicz. et al. Adaptive remeshing for compressible flow Computation[J]. Journal of Computational Physics, 1987,72:449 466.

[4] EYMARD R. Mathematical and numerical properties of control-volume finite-element scheme for reservoir simulation [J].SPE Reservoir Engineering,November,1994:283 289.

[5] Barry Joe. Construction of three-dimension Delaunay triangulations using local transformations[J].Comput. Aided Gemetric Design,1991,8:123 142.

[6] JOE B.Delaunay triangular meshes in convex polygons[J].SIAM J.Sci Stat.Com,1986,7:514 539.

[7] WEATHERILL $\mathrm{N}$ P. A method for generating irregular computational grids in multiply connected planar domains[J].International Journal for numerical methods in fluids, 1988,8:181 197.

[8] Li Wenwu, Ji Changming, You Wenxia. Study of Isohyets Generation Algorithm in Reservoir Dispatch Automation System. Journal of Wuhan University,4 (2006),p46 48

[9] Huang Binghu,Sun Genyun.Analysis and realization of automatic extracting isohyets.Computer Engineering and Design,2010,31(15),p426 613 Design,2010,31(15),p426 613

[10] Peng Siling, Deng Min, Drawing Rain Isolines Based on ArcEngine. Geomatics World,2010,p426 613 